\section{Effect of Ionic Strength on Activation of Prophenoloxidase in Its Crude Solution}

\author{
Takashi InABa, * Genshin NAmiHIRA, ${ }^{* 2}$ \\ Michihisa KAWANO*3 and \\ Masaru FunATSU*4 \\ Laboratory of Biochemistry, Faculty of \\ Agriculture, Kyushu University, \\ Fukuoka 812, Japan
}

Received July 10, 1978

We reported previously that protyrosinase in prepupae of housefly, Musca domestica vicina Maquart, could be activated either by anionic detergents or by its natural activator extracted from aged pupae. ${ }^{1 \sim 3}$ ) This enzyme was later demonstrated by Karlson et al. ${ }^{4}$ ) to be phenoloxidase ( $o$-Diphenol: oxygen oxidoreductase, EC 1.10.3.1).

Preliminary experiments on prophenoloxidase also showed that it was activated autocatalytically during dialysis against deionized water. The autocatalytic activation was thought to be of significance in connection not only with the purification of prophenoloxidase but also with the chemical nature of the proenzyme.

Prophenoloxidase extracted from prepupae of housefly, $M$. domestica vicina Maquart, with $0.2 \mathrm{M}$ phosphate buffer, $\mathrm{pH}$ 6.0, was kept intact in the limited range below $\mathrm{pH} 6.0$, whereas at $\mathrm{pH}$ higher than 7.0 it was activated autocatalytically, as shown in Fig. 1. This finding suggests that a change in the charge of prophenoloxidase is involved in the activation. The phenomenon is similar to that in the report of Allen et $a l^{5)}$ who found that protyrosinase in the extract of eggs of grasshopper, Melanoplus differentialis, was activated autocatalytically at pH 9.3 or above. However, no mechanism has been suggested to account for the autocatalytic activation due to the change in $\mathrm{pH}$ of the solution. On the other hand, the prophenoloxidase was irreversibly denatured during dialysis against $0.2 \mathrm{M}$ phosphate buffer of pH 5.5 or below.

It was found that the autocatalytic activation of prophenoloxidase also occurred in a low concentra-

Present addresses: * Laboratory of Biological Chemistry, Faculty of Agriculture, Saga University, Saga. *2 Laboratory of Food Chemistry, Department of Food and Nutrition, Nakamura Gakuen College. *a Kumiai Chemical Institute, Shizuoka. ** Department of Applied Microbial Technology, The Kumamoto Institute of Technology, Kumamoto.

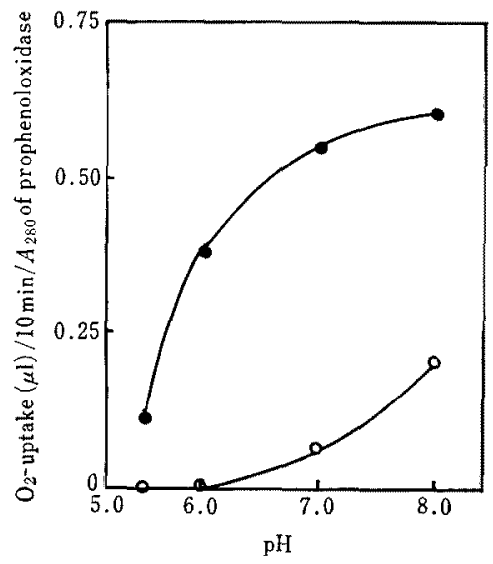

Frg. 1. Effect of $\mathrm{pH}$ on Activation of Prophenoloxidase.

The crude prophenoloxidase solution was dialyzed against $0.2 \mathrm{M}$ phosphate buffer of different $\mathrm{pHs}$ for $24 \mathrm{hr}$ at $3{ }^{\circ} \mathrm{C}$. The change in volume of oxygen uptaken due to the oxidation of catechol catalyzed by phenoloxidase was measured at $25^{\circ} \mathrm{C}$ with a Warburg manometer according to the procedures in the preceding paper. ${ }^{1}$

$0-0$, prophenoloxidase; -0 , prophenoloxidase + natural activator.

tion of buffer even at $\mathrm{pH}$ 6.0. Prophenoloxidase in the crude solution was dialyzed against $0.01,0.05,0.1$ and $0.2 \mathrm{M}$ sodium acetate buffer, $\mathrm{pH} 6.0$, for $24 \mathrm{hr}$ at $3^{\circ} \mathrm{C}$, and then the phenoloxidase activity in the dialyzed solution was measured both in the presence and in the absence of natural activator. The natural activator was obtained from the aged pupae of housefly according to the method described in the preceding paper. ${ }^{33}$ As shown in Fig. 2, prophenoloxidase was kept intact only in $0.2 \mathrm{M}$ buffer without any autocatalytic activation, while at a concentration of lower than $0.2 \mathrm{M}$ it was activated autocatalytically. In $0.2 \mathrm{M}$ buffer, however, prophenoloxidase was activated only by the natural activator. These results show that prophenoloxidase is not irreversibly denatured in $0.2 \mathrm{M}$ buffer but is in an inactive form.

A similar tendency concerning salt concentrations of buffer was observed also in phosphate buffer. In this buffer, prophenoloxidase was kept intact at concentrations of 0.15 and $0.2 \mathrm{M}$ and was activated autocatalytically at $0.1 \mathrm{M}$ or below.

In addition, prophenoloxidase in the crude solution was dialyzed against $0.01 \mathrm{M}$ phosphate buffer, $\mathrm{pH} 6.0$, containing $0.01,0.05$ and $0.1 \mathrm{M} \mathrm{NaCl}$. At the $\mathrm{NaCl}$ concentration of $0.1 \mathrm{M}$, prophenoloxidase was kept stable without any autocatalytic activation.

From these facts it was inferred that the autocatalytic activation of prophenoloxidase depends upon the 


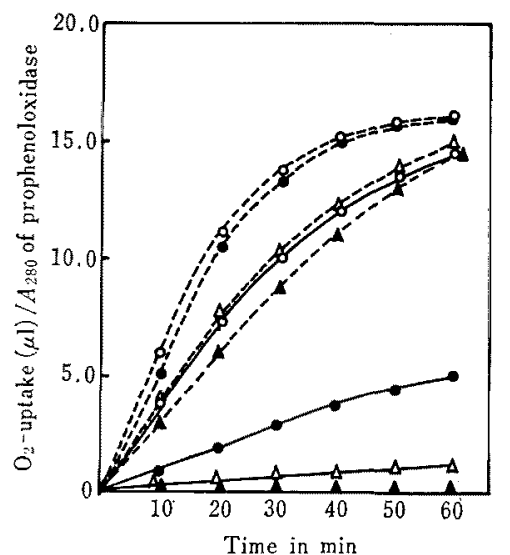

FIG. 2. Autocatalytic Activation of Prophenoloxidase Depending upon Change in Concentration of Sodium Acetate Buffer.

The crude prophenoloxidase solution was dialyzed against different concentrations of sodium acetate buffer, pH 6.0 , for $24 \mathrm{hr}$ at $3^{\circ} \mathrm{C}$.

$\bigcirc-0,0.01 \mathrm{M}$ buffer; $0-0,0.01 \mathrm{M}$ buffer + natural activator; - $0.05 \mathrm{M}$ buffer; --.- $0.05 \mathrm{M}$ buffer + natural activator; $\triangle-\triangle, 0.10 \mathrm{M}$ buffer; $\triangle--\triangle, 0.10 \mathrm{M}$ buffer + natural activator; $\boldsymbol{\Delta}-\boldsymbol{\Lambda}, 0.20 \mathrm{M}$ buffer; $\mathbf{\Delta}---\boldsymbol{\Delta}$, $0.20 \mathrm{M}$ buffer + natural activator.

salt concentration or ionic strength of the solution. This seems to be supported also by the following finding. Prophenoloxidase in the crude solution was dialyzed against $0.001 \mathrm{M}$ phosphate buffer, $\mathrm{pH} 6.0$, overnight at $3^{\circ} \mathrm{C}$ at the first step. At the second step, the above solution was further dialyzed against $0.2 \mathrm{M}$ phosphate buffer of the same pH. At the third step, the solution from the second step was dialyzed under the same conditions as the first step. The phenoloxidase activity in each solution was determined both in the presence and in the absence of the natural activator. During the first dialysis prophenoloxidase was activated autocatalytically. However, after the second dialysis, although no phenoloxidase activity was detected in the absence of natural activator, the complete activity of phenoloxidase was observed in its presence. In the second dialysis, phenoloxidase formed during the first dialysis appeared to be converted to prophenoloxidase, which was then activated again in the third dialysis. Thus, the activation of prophenoloxidase is reversible depending upon the salt concentration or ionic strength of the solution.

There is another possibility for the activation of prophenoloxidase in the solution of a low salt concentration. Namely, lowering of osmotic pressure of the solution might cause the activation. In order to examine this possibility, prophenoloxidase in the crude solution was dialyzed against $0.05,0.1,0.5$ and $1.0 \mathrm{M}$ sucrose in $0.01 \mathrm{M}$ phosphate buffer, $\mathrm{pH} 6.0$, and the resulting phenoloxidase activity of each solution was determined. As the result, all of the concentrations of sucrose tested could not restrict the activation of prophenoloxidase. Therefore, the activation of prophenoloxidase occurs independently from the lowering of osmotic pressure of its solution. This finding may exclude the possibility that phenoloxidase is released by destruction of the granules binding phenoloxidase under lower osmotic pressure, as noted by Karlson et al. ${ }^{4)}$ and shows that prophenoloxidase in question is a soluble proenzyme.

\section{REFERENCES}

1) M. Funatsu and T. Inaba, Agric. Biol. Chem., 26, 536 (1962).

2) T. Inaba, Y. Suetake and M. Funatsu, ibid., 27, 332 (1963).

3) T. Inaba and M. Funatsu, ibid., 28, 206 (1964).

4) P. Karlson and H. Lieben, $Z$. Physiol. Chem., 326, 135 (1961).

5) T. H. Allen, A. B. Otis and J. H. Bodine, J, Gen. Physiol., 26, 151 (1943). 